\title{
Hematopoietic neoplasms with 9p24/JAK2 rearrangement: a multicenter study
}

\author{
Guilin Tang ${ }^{1} \cdot$ John Kennedy Sydney Sir Philip ${ }^{2} \cdot$ Olga Weinberg $^{3} \cdot$ Wayne Tam $^{4} \cdot$ Sam Sadigh ${ }^{5}$. Jonathan I. Lake ${ }^{5}$ \\ Elizabeth M. Margolskee $\mathbb{D}^{4} \cdot$ Heesun J. Rogers ${ }^{6} \cdot$ Roberto N. Miranda ${ }^{1}$. Carlos Bueso-Ramos $\mathrm{C}^{1} \cdot$ Eric D. Hsi ${ }^{6}$. \\ Attilio Orazi ${ }^{4} \cdot$ Robert P. Hasserjian ${ }^{7} \cdot$ Daniel A. Arber ${ }^{2} \cdot$ Adam Bagg $^{5} \cdot$ Sa A. Wang ${ }^{1}$
}

Received: 15 August 2018 / Revised: 28 September 2018 / Accepted: 9 October 2018 / Published online: 6 November 2018

(c) United States \& Canadian Academy of Pathology 2018

\begin{abstract}
The purpose of this study is to examine hematopoietic neoplasms with 9p24/JAK2 rearrangement including neoplasms associated with $\mathrm{t}(8 ; 9)(\mathrm{p} 22 ; \mathrm{p} 24) / P C M 1-J A K 2$ fusion neoplasm as well as cases with translocations involving 9p24/JAK2 and other partner genes. From seven large medical centers, we identified ten patients with $\mathrm{t}(8 ; 9)(\mathrm{p} 22 ; \mathrm{p} 24) / P C M 1-J A K 2$ and 3 with $\mathrm{t}(9 \mathrm{p} 24 ; \mathrm{v}) / J A K 2$ at diagnosis. Majority of the cases showed myeloproliferative neoplasm (MPN) associated features $(n$ $=7$ ) characterized by variable degrees of eosinophilia, myelofibrosis, frequent proliferations of early erythroblasts in bone marrow and extramedullary sites, and infrequent/absent somatic mutations. Other less common presentations included myelodysplastic syndromes (MDS) or MDS/MPN (one each). Four patients presented with B-lymphoblastic leukemia (BALL), and of them, two patients with $\mathrm{t}(8 ; 9)(\mathrm{p} 22 ; \mathrm{p} 24.1)$ were proven to be B-lymphoblastic crisis of MPN; and the other two cases with $\mathrm{t}(9 \mathrm{p} 24 ; \mathrm{v})$ both were de novo B-ALL, BCR-ABL1-like (Ph-like). We show that the hematopoietic neoplasms with 9p24/JAK2 rearrangement are extremely rare, and most of them are associated with $\mathrm{t}(8 ; 9)(\mathrm{p} 22 ; \mathrm{p} 24) / P C M 1-J A K 2$, a recent provisional World Health Organization entity under "myeloid/lymphoid neoplasm with a specific gene rearrangement". Cases of $\mathrm{t}(8 ; 9)(\mathrm{p} 22 ; \mathrm{p} 24) / P C M 1-J A K 2$, though heterogeneous, do exhibit some common clinicopathological characteristic features. Cases with $\mathrm{t}(9 \mathrm{p} 24 ; \mathrm{v}) / J A K 2$ are extremely rare; while such cases with a MPN presentation may resemble $\mathrm{t}(8 ; 9)(\mathrm{p} 22$; p24.1)/PCM1-JAK2, B-ALL cases presenting de novo B-ALL might belong to Ph-like B-ALL.
\end{abstract}

Electronic supplementary material The online version of this article (https://doi.org/10.1038/s41379-018-0165-9) contains supplementary material, which is available to authorized users.

Sa A. Wang

Swang5@mdaderson.org

1 Department of Hematopathology, The University of Texas M.D. Anderson Cancer Center, Houston, TX, USA

2 Department of Pathology, University of Chicago, Chicago, IL, USA

3 Department of Pathology, Boston Children Hospital, Boston, MA, USA

4 Department of Pathology and Laboratory Medicine, Weill Cornell Medical College, New York, NY, USA

5 Department of Pathology and Laboratory Medicine, University of Pennsylvania, Philadelphia, PA, USA

6 Department of Laboratory Medicine, Cleveland Clinic, Cleveland, $\mathrm{OH}$, USA

7 Department of Pathology, Massachusetts General Hospital, Boston, MA, USA

\section{Introduction}

JAK2 is a cytoplasmic tyrosine kinase that activates multiple downstream transducers through the JAK-STAT pathway. JAK2 plays a central role in hematopoiesis, cell proliferation and differentiation [1]. JAK2 V617F mutation leads to constitutive activation of JAK2 and is present in approximately $95 \%$ cases of polycythemia vera and 50 60\% essential thrombocythemia and primary myelofibrosis. Ruxolitinib, a JAK2 inhibitor, has been approved by the United States Food and Drug Administration for the treatment of intermediate- or high-risk myelofibrosis and has shown efficacy in improving systemic symptoms and splenomegaly in those patients [2].

Chromosomal translocations involving 9p24/JAK2 have been reported with various partner genes [3]. $\mathrm{t}(8 ; 9)(\mathrm{p} 22$; p24.1) was first reported in 1990 in patients with "Philadelphia chromosome $(\mathrm{Ph})$-negative neutrophilic myelofibrosis" [4], and this cytogenetic abnormality was shown to result in PCM1-JAK2 fusion in 2005 [5]. To date, 40 cases 
of lymphoid/myeloid neoplasms associated with $\mathrm{t}(8 ; 9)(\mathrm{p} 22$; p24.1)/PCM1-JAK2 have been reported [3, 6]. In 2014, Bain and colleagues [3] summarized 33 cases of $t(8 ; 9)(\mathrm{p} 22$; p24.1) that had been published in literature as well as cases of JAK2 rearrangement with other variant partner genes, such as $\mathrm{t}(9 ; 12)(\mathrm{p} 24.1 ; \mathrm{p} 13) / E T V 6-J A K 2$ and $\mathrm{t}(9 ; 22)(\mathrm{p} 24.1$; $\mathrm{q} 11.2) / B C R-J A K 2$.

These neoplasms presented with an array of disease entities, including Ph-negative myeloproliferative neoplasms (MPN); chronic eosinophilic leukemias (CEL), myelodysplastic/ myeloproliferative neoplasms (MDS/ MPN), MDS, acute myeloid leukemia (AML), B- and Tlymphoblastic leukemia (ALL) or, rarely, B- or T-cell lymphomas. Although clinically heterogeneous, these neoplasms with $\mathrm{t}(8 ; 9)(\mathrm{p} 22 ; \mathrm{q} 24.2) / P C M 1-J A K 2$ appeared to share some common features, including frequent eosinophilia, aberrant overexpression of the cytokine receptor JAK2 as a result of a gene fusion, and the potential to involve myeloid, lymphoid or both lineages. Their clinical and genetic characteristics were considered to be similar to those seen in the group of diseases termed by the World Health Organization (WHO) 2008 as myeloid/lymphoid neoplasms with eosinophilia and PDGFRA, PDGFRB, and FGFR1 rearrangements. Thus, in 2017 the WHO Classification, $t(8 ; 9)(p 22 ; p 24) / P C M 1-J A K 2$ is added to the category of Myeloid/lymphoid neoplasms with eosinophilia and gene rearrangement as a provisional entity [3, 7]. Cases in which $J A K 2$ was rearranged with other partner genes such as $\mathrm{t}(9 ; 12)(\mathrm{p} 24.1 ; \mathrm{p} 13.2) / E T V 6-J A K 2$ and $\mathrm{t}(9 ; 22)(\mathrm{p} 24.1$; q11.2)/BCR-JAK2 were postulated to represent possible variants of this provisional entity.

Due to the rarity of these diseases, we conducted the current multicenter study as an effort of the Bone Marrow Pathology Group to better understand the spectrum of hematopoietic neoplasms with 9p24/JAK2 rearrangement, including their pathological characteristics and the natural history of these neoplasms.

\section{Methods}

\section{Patients}

A search was set to find hematopoietic neoplasms with chromosome 9p24 abnormalities and/or JAK2 rearrangement that were not lymphoma or myeloma. The cases were collected from MD Anderson Cancer Center, Brigham and Women's Hospital, University of Chicago, Massachusetts General Hospital, Cleveland Clinic, the Hospital of the University of Pennsylvania, and Weill Cornell Medical Center. The study periods ranged from 8 to 14 years among the participating institutions, with a median of 10 years. Clinical information and follow-up data were retrieved from the electronic medical records. This study was approved by the Institutional Review Boards of all participating institutions.

\section{Chromosomal analysis and fluorescence in situ hybridization (FISH)}

Conventional chromosomal analysis was performed on Gbanded metaphases prepared from unstimulated 24-h and 48-h BM aspirate cultures using standard techniques. Twenty metaphases were analyzed and the results were reported using the 2016 International System for Human Cytogenetics Nomenclature (ISCN 2016). A complex karyotype was defined as $\geq 3$ chromosomal abnormalities.

FISH analysis was performed on BM aspirate smears, cultured (for cytogenetics) BM cells or paraffin-embedded lymph node tissue section with a JAK2 dual-color breakapart probe (Cytocell, Inc., Tarrytown, NY) or a PCM1$J A K 2$ dual-color dual fusion probe set (Empire Genomics LLC., Buffalo, NY). Combined morphology and FISH analysis was performed on Wright-Giemsa stained BM aspirate smears with JAK2 FISH probes as described previously [8].

\section{Morphologic and histological assessment}

Wright-Giemsa stained peripheral blood (PB) and bone marrow (BM) aspirate smears and H\&E-stained BM aspirate clot and core biopsy tissue sections were reviewed. The PB smear and BM biopsy section, aspirate smears were assessed for eosinophils, blast percentages, BM cellularity, myeloid:erythroid ratio, megakaryocyte numbers and distribution, and morphologic dysplasia of all lineages. Myelofibrosis was evaluated by reticulin and trichrome stains performed on the BM core biopsy when available. The grade of myelofibrosis was based on the European Consensus on grading of BM fibrosis [9]. For cases with extramedullary involvement, the tissue samples were evaluated.

\section{Immunophenotyping}

Flow cytometry immunophenotyping was performed at each institution for cases with increased blasts. Basic markers for leukemic phenotyping were assessed at all institutions, including CD34, CD117, CD13, CD33, HLADR, CD19, CD10, and CD45. For cases in which the JAK2 rearrangement was acquired at disease relapse, the immunophenotype of the blasts at original diagnosis and at relapse were compared. In some cases, immunohistochemistry including CD34, CD117, MPO, lysozyme, and glycophorin A was performed to characterize the blasts in biopsy material. 
Fig. 1 Cytogenetic findings on case \#10. a chromosomal analysis showed a karyotype of 46,XX,t(8;9)(p22;p24.1); b FISH analysis using $J A K 2$ dualcolor break-apart probe shows a split signal pattern $(1$ red 1 green, marked by arrow) consistent with $J A K 2$ rearrangement; c FISH analysis with $P C M 1$ (green)/JAK2 (red) dual-color dual fusion probe shows 2 fusion (yellow, marked by arrow) signals, consistent with $P C M-J A K 2$ rearrangement A 3

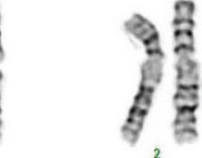
ll 6. 焉 8 है $\frac{8}{2}$ 6. है है 量是 量 कृ 현 8

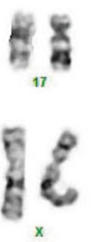

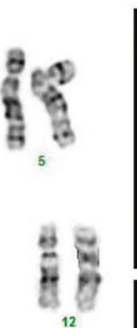
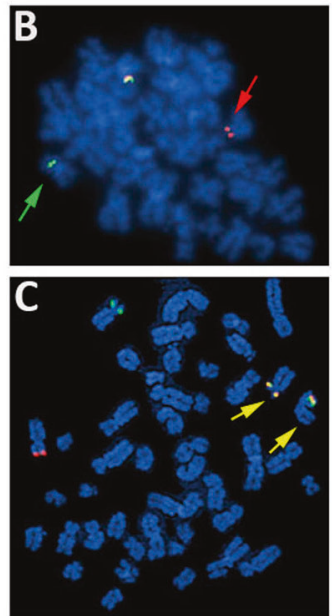

\section{Targeted next-generation sequencing (NGS)}

Targeted NGS studies using panels of genes commonly altered in myeloid neoplasia were performed in six patients. Three patients had NGS performed as part of the clinical workup (case \#5 and \#7: 147 gene panel; case \#10: 81 gene panel); three patients (cases \#1, 6, 9) had NGS performed on fresh-frozen bone marrow DNA samples with a 45-gene panel using the same method as described previously [10, 11]. The gene panel information is summarized in the Supplementary Information.

\section{Results}

\section{Patients}

A total of 29 patients with 9p24 abnormalities were found, including 14 with $\mathrm{t}(8 ; 9)(\mathrm{p} 22 ; \mathrm{p} 24.1)$ and 15 with other 9p24 abnormalities. The latter group included 13 patients with $t$ $(9 \mathrm{p} 24 ; \mathrm{v})$ other than $\mathrm{t}(8 ; 9)(\mathrm{p} 22 ; \mathrm{p} 24.1)$ and two with add(9) (p24). FISH using a JAK2 dual-color break-apart probe was performed in all these 15 patients and revealed a $J A K 2$ rearrangement only in four $(4 / 15,27 \%)$ patients, encompassing one each of $\mathrm{t}(9 ; 22)(\mathrm{p} 24.1 ; \mathrm{q} 11.2), \mathrm{t}(5 ; 9)(\mathrm{q} 14 ; \mathrm{p} 24.1)$, $\mathrm{t}(5 ; 9)(\mathrm{q} 11.2 ; \mathrm{p} 24.1)$, and add(9)(p24). The 11 cases with 9p24 abnormalities but lacking JAK2 rearrangement are listed in the Supplementary Table and excluded from this study. FISH was performed in $11 / 14$ cases with $\mathrm{t}(8 ; 9)$ (p22; p24.1) and positive for JAK2 rearrangement in all 11 (11/ $11,100 \%)$. FISH with PCM1-JAK2 dual-color dual fusion probes was attempted on $\mathrm{BM}$ aspirate smears, but was unsuccessful; however, we were able to successfully perform it on two cases with available culture cells and confirmed PCM1-JAK2 fusion in both patients. To focus this study to cases with $J A K 2$ rearrangement detected at the time of diagnosis, we further excluded five cases that 9p24/JAK2 rearrangements were detected at disease relapse. The final included cases were 10 with $\mathrm{t}(8 ; 9)(\mathrm{p} 22 ; \mathrm{p} 24.1)$ and three patients with $J A K 2$ rearranged with other partner genes.

\section{Patients with $\mathrm{t}(8 ; 9)(\mathrm{p22} ; \mathrm{p24.1)/PCM1-JAK2}$ rearrangement}

Of the ten patients with $\mathrm{t}(8 ; 9)(\mathrm{p} 22 ; \mathrm{p} 24.1) / P C M 1-J A K 2$ (Fig. 1a), FISH using a JAK2 dual-color break-apart probe (Fig. 1b) was performed in seven cases with material available, including five on direct BM aspirate smears, one on culture cells, and one on lymph node paraffin-embedded slide, and confirmed the presence of JAK2 rearrangement in all seven patients (100\%) (Tables 1 and 2). Due to a high concordance of $\mathrm{t}(8 ; 9)(\mathrm{p} 22 ; \mathrm{p} 24.1)$ with JAK2 rearrangement in our study and in published cases [3], the other three cases with $\mathrm{t}(8 ; 9)(\mathrm{p} 22 ; \mathrm{p} 24.1)$ that lacked material to confirm JAK2 rearrangement were retained in the study. FISH with PCM1-JAK2 dual-color dual fusion probes showed PCM1$J A K 2$ fusion in patient \#10 (Fig. 1c).

Of the ten patients with $\mathrm{t}(8 ; 9)(\mathrm{p} 22 ; \mathrm{p} 24.1)$, five patients $(50 \%)$ had a diagnosis of MPN in chronic phase, one MPN in accelerated/blast phase, one MDS, one CMML and two B-ALL. The five cases (\#1-5) of MPN in chronic phase shared a number of features (Tables 1 and 2). All five patients presented with splenomegaly and three patients additionally had documented lymphadenopathy. At the initial presentation, variable degrees of $\mathrm{PB}$ eosinophilia were present, ranging from 6 to 39\% (median 13\%), with a median absolute count of 864/ $\mu \mathrm{L}$ (range 366--3315); however, only one patient had an absolute eosinophils > 1500/ $\mu \mathrm{L}$ (hypereosinophilia). In patient \#1 and \#2, PB eosinophilia started as mild and later became prominent (8-23\% and 6-23\%, respectively). There was no PB basophilia (median 0\%, 0-2\%); no monocytosis (median 


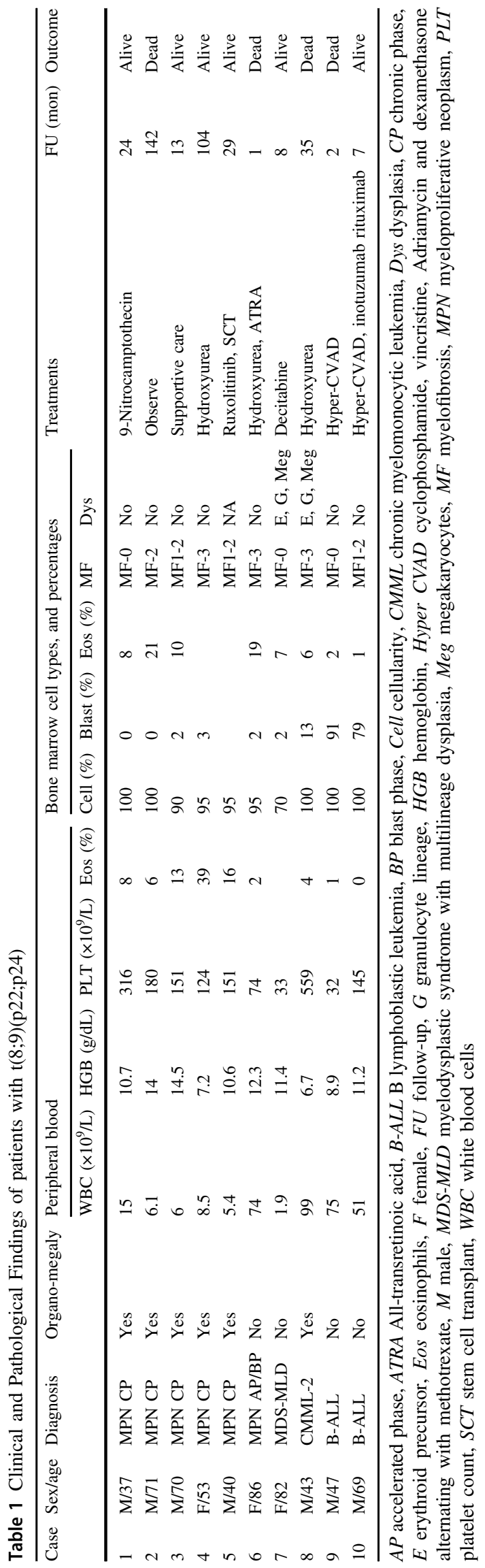

5\%, 4-7\%), no left-shifted myeloid cells (myelocytes/ metamyelocytes/promyelocytes, median 0\%, 0-2\%), and no circulating blasts $(0 \%)$. All five patients showed a hypercellular BM (90-100\%), with increased eosinophils, and frequent myelofibrosis (MF1-3 in 4/5 patients) (Fig. 2). Myeloid:erythroid ratio was difficult to estimate due to hemodilution of BM aspirates, but overall increased. Eosinophils showed various morphological features, some unremarkable; but hypogranulation, abnormal eosinophilic/ basophilic granulation, hypersegmentation, immature forms, were frequently observed (Fig. 2c). Megakaryocytes varied in number, but were morphologically unremarkable in all five patients (Figs. 2d and 3a). Immature erythroid precursors in large aggregates or nodules were observed in the BM biopsies of four patients (patient 1,2,3, and 5) (Figs. 2a, 2b and 3a). However, dyserythropoiesis was not significant in any of the cases. There was no significant dysgranulopoiesis (Fig. 2c). Three patients had lymph node biopsied and one also had a splenectomy. The lymph nodes and spleen all exhibited extramedullary disease infiltrate with large clusters or aggregates of immature erythroid precursors, raising the concern of extramedullary pure erythroid leukemia in patient 5 (Fig. 3b). None of the cases had JAK2 V617F mutation. NGS was performed in two patients (cases \#1 and 5) and showed no mutations. Regarding treatment, only one patient received ruxolitinib followed by hematopoietic stem-cell transplant (HSCT), while the other patients were only treated with hydroxyurea, low intensity chemotherapy (9-Nitrocamptothecin), or received supportive care. These five patients showed no disease progression during the follow-up period (median follow-up 29 months, range 13-142 months).

Patient \#6 presented with marked leukocytosis, with leftshifted granulocytic cells and numerous normal-appearing promyelocytes (48\%) but only $2 \%$ eosinophils in the PB. The BM showed a markedly left-shifted myeloid maturation with increased eosinophils (19\%) and MF3 fibrosis. No Auer rods were identified in the PB or BM. PML-RARA was detected by FISH at a very low level (1.5\% in PB and $1 \%$ in $\mathrm{BM})$. The patient was treated with all trans retinoic acid (ATRA) and arsenic trioxide (ATO), however the posttreatment BM showed persistent disease and the patient died 1 month later due to disease progression. NGS showed no mutations. The case was difficult to classify, but most compatible with an MPN, unclassifiable in either accelerated or blast phase.

Patient \#7 presented with pancytopenia and the BM showed trilineage dyspoiesis and $2 \%$ blasts, and was diagnosed with MDS with multilineage dysplasia. There were small clusters of immature erythroid precursors. He was treated with decitabine and alive at 8 months of follow-up. Patient \#8 had an outside diagnosis of MDS/MPN, consistent with CMML-1 and received hydroxyurea. At the 
Table 2 Cytogenetics and molecular findings of patients with $\mathrm{t}(8 ; 9)(\mathrm{p} 22 ; \mathrm{p} 24)$

\begin{tabular}{llll}
\hline Cases & Karyotype & FISH for JAK2 & Molecular mutations \\
\hline 1 & $46, X Y, t(8 ; 9)(\mathrm{p} 22 ; \mathrm{p} 24)[20]$ & Positive & Negative: All genes (NGS) \\
2 & $46, \mathrm{XY}, \mathrm{t}(8 ; 9)(\mathrm{p} 22 ; \mathrm{p} 24)[19]$ & Positive & Negative: JAK2 \\
3 & $46, \mathrm{XY}, \mathrm{t}(8 ; 9)(\mathrm{p} 21 ; \mathrm{p} 24)[13] / 46, \mathrm{XY}[7]$ & $\mathrm{ND}$ & $\mathrm{ND}$ \\
4 & $46, \mathrm{XX}, \mathrm{t}(8 ; 9)(\mathrm{p} 22 ; \mathrm{p} 24)[17] / 46, \mathrm{XX}[3]$ & Positive & ND \\
5 & $46, \mathrm{XY}, \mathrm{t}(8 ; 9)(\mathrm{p} 22 ; \mathrm{p} 24)[20]$ & Positive & Negative: All genes (NGS) \\
6 & $46, \mathrm{XX}, \mathrm{t}(8 ; 9)(\mathrm{p} 22 ; \mathrm{p} 24)[20]$ & Positive & Negative: All genes (NGS) \\
7 & $46, \mathrm{XX}, \mathrm{t}(8 ; 9)(\mathrm{p} 22 ; \mathrm{p} 24)[20]$ & $\mathrm{ND}$ & Positive: ASXL1, RUNX1, SRSF2, \\
& & & TET2 \\
& & & Negative: all others (NGS) \\
8 & $46, \mathrm{XY}, \mathrm{t}(8 ; 9)(\mathrm{q} 22 ; \mathrm{p} 24)[20]$ & ND & ND \\
9 & $46, \mathrm{XY}, \mathrm{t}(8 ; 9)(\mathrm{p} 22 ; \mathrm{p} 24)[1] / 47, \mathrm{idem},+21$ & Positive & Positive: ETV6, TP53 \\
& {$[19]$} & & Negative: all others (NGS) \\
10 & $45, \mathrm{XY},-7, \mathrm{t}(8 ; 9)(\mathrm{p} 22 ; \mathrm{p} 24)[15] / 45 \sim 46, i d e m$ & Positive & Positive: ASXL1, TET2, BCOR \\
& {$[\mathrm{cp} 5]$} & & Negative: all others (NGS) \\
\hline
\end{tabular}

$N D$ not done, $N G S$ next generation sequencing time of the examined biopsy, the patient had a high WBC $\left(99 \times 10^{9} / \mathrm{L}\right)$, monocytosis $\left(9.9 \times 10^{9} / \mathrm{L}\right)$, and $6 \%$ blasts, hepatosplenomegaly, BM with MF3 fibrosis and $13 \%$ blasts, compatible with CMML-2. He continued treatment with hydroxyurea but progressed to AML and died 35 months from the initial CMML diagnosis. In both patients, there was no significant peripheral eosinophilia, but both BM aspirate smears showed mildly increased eosinophils (7\% and $6 \%$ respectively). NGS showed ASXL1, RUNX1, SRSF2, and TET2 mutations in patient \#7; mutational testing was not performed in patient \#8.

Two patients (\#9 and \#10) presented with de novo BALL, with no documented history of MPN. Both patients had $>80 \%$ circulating B-lymphoblasts with no eosinophilia. The BM showed numerous blasts $(79 \%$ and $91 \%$ respectively) without significant eosinophilia or fibrosis. The blasts in both cases had a common-B immunophenotype, CD34+, CD19+, CD10+, CD20 partial+, and TDT + . NGS showed ETV6 and TP53 mutations in case \#9 and ASXL1, TET2, and BCOR mutations in patient \#10. Patient \#9 was treated with hyper-CVAD (cyclophosphamide, vincristine, doxorubicin). The follow-up BM performed on the day 21 BM showed an $80 \%$ cellularity with $28 \%$ blasts. Eosinophils were not increased. A combined morphology and FISH analysis performed on day $21 \mathrm{BM}$ smear detected $J A K 2$ rearrangement in $60 \%$ of cells, involving not only blasts but also granulocytes and erythroid elements (Fig. 4a, 4b). Patient \#9 died of sepsis 2 months after the initial diagnosis. Patient \#10 was treated with hyper-CVAD plus inotuzumab and rituximab. The patient achieved morphological remission at day 7 , but showed persistent minimal residual disease (MRD) by flow cytometry at all later time points. Leukocytosis without circulating B-lymphoblasts was noted on several occasions, with WBC up to $39.4 \times$ $10^{9} / \mathrm{L}$ with mainly of neutrophils. At the last follow-up
(7 months after diagnosis), while the patient was continuing treatment with inotuzumab plus low intensity chemotherapy, his BM showed hypercellularity with some eosinophil precursors (Fig. 4c, 4d), and a positive MRD $(0.13 \%)$ by flow cytometry. FISH revealed JAK2 rearrangement in 82/ $200(41 \%)$ of cells, including many segmented cells (neutrophils) (Fig. 4e). These findings suggested a lymphoblastic crisis of an underlying chronic myeloid neoplasm in both cases. The background chronic myeloid neoplasms were difficult to subclassify either due to early patient demise or the patient being in the course of chemotherapy.

\section{JAK2 rearrangement with variant partner genes}

Three patients with variant translocations were identified. These included one each of $\operatorname{der}(9) \mathrm{t}(9 ; 22)(\mathrm{p} 24.1 ; \mathrm{q} 11.2)$ (patient \#11), t(5;9)(q14;p24.1) (patient \#12), and $\mathrm{t}(5 ; 9)$ (q11.2;p24.1) (patient \#13). Patient \#11 presented with leukocytosis with $10 \%$ circulating eosinophils, and hepatosplenomegaly and was diagnosed with CEL, NOS. Karyotype showed $\operatorname{der}(9) \mathrm{t}(9 ; 22)(\mathrm{p} 24.1 ; \mathrm{q} 11.2)$ and FISH showed JAK2-BCR rearrangement. The patient received HSCT three months after diagnosis and remained disease free at the last follow-up (39 months after diagnosis).

Two patients (\#12 and \#13) presented with B-ALL, with $J A K 2$ rearrangements detected at the time of initial diagnosis. Both patients had a very high WBC with a large number of circulating blasts and no eosinophilia. Blasts had a common B immunophenotype, CD34+, CD19+, CD10 + , CD20 partial+, TDT + . Both patients were treated with induction chemotherapy and achieved morphological remission; however, MRD was not assessed. At the time of remission, patient \#12 showed no persistent JAK2 rearrangement by FISH and patient \#13 showed a normal karyotype without evidence of the previously detected 46,XY,t 
Fig. 2 Bone marrow (BM) morphological findings of case \#2 (myeloproliferative neoplasm, chronic phase). a (×10) hypercellular BM with overall decreased

megakaryocytes and a small erythroid island; $\mathbf{b}(\times 40)$ the immature erythroid island shows brisk mitosis, and the adjacent area shows many eosinophils and neutrophils; $\mathbf{c}(\times 100)$ the $\mathrm{BM}$ aspirates are often hemodilute due to underlying fibrosis. There is no significant dyserythropoiesis or dysgranulopoiesis. Eosinophils are increased, some exhibiting abnormal nuclear segmentation; d $(\times 40)$ stromal edema may be seen. There is no significant dysmegakaryopoiesis
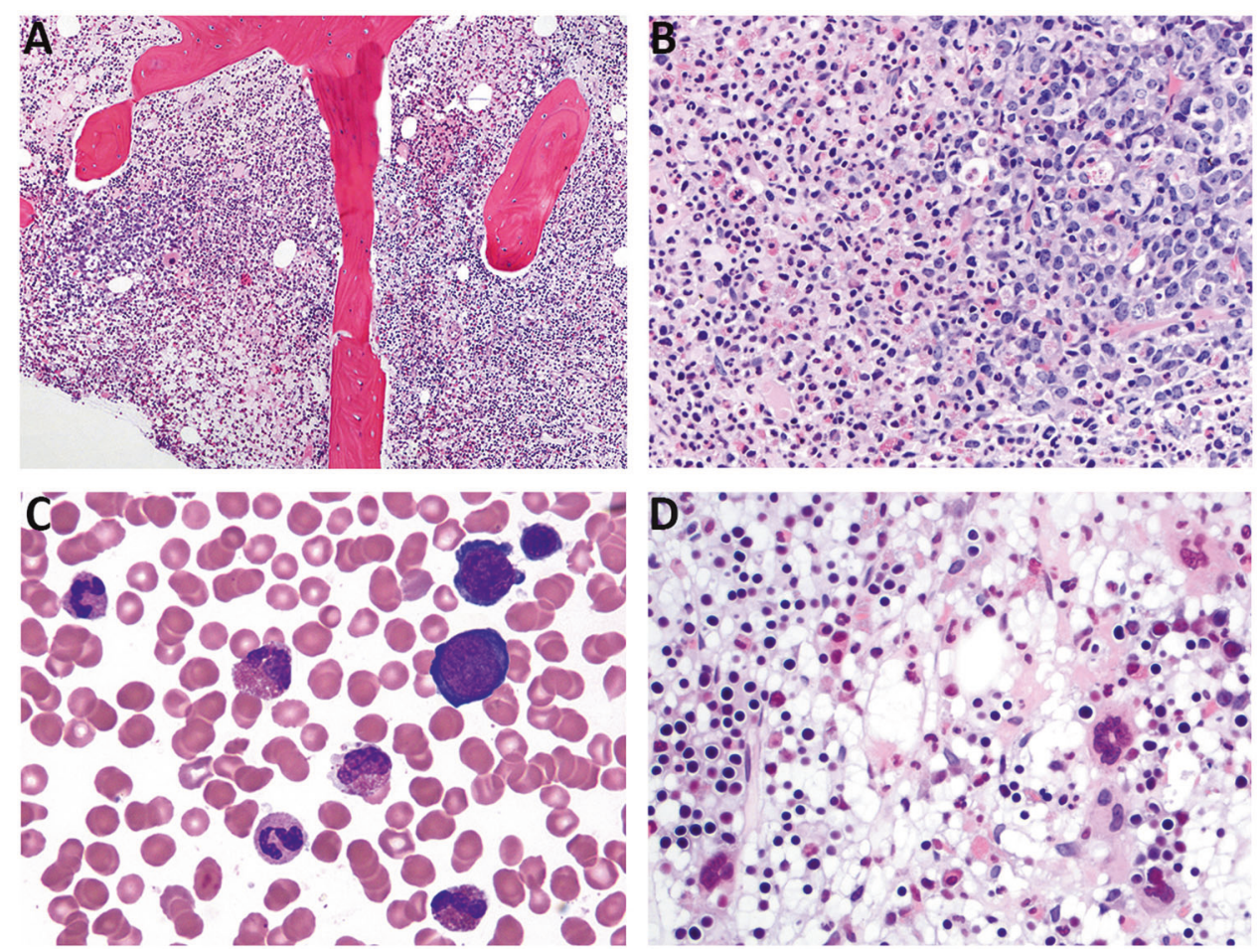

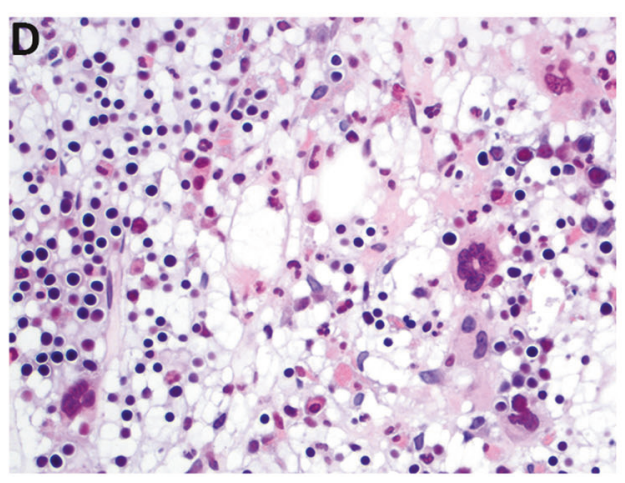

(5;9)(q11.2;p24.1). This indicated that JAK2 rearrangement was limited to B-lymphoblasts not in the background myeloid and erythroid cells.

\section{Discussion}

This is the largest single series of hematopoietic neoplasms with 9p24/JAK2 rearrangement reported to date, including ten cases with $\mathrm{t}(8 ; 9) / P C M 1-J A K 2$ and three in which JAK2 was arranged with other partner genes. Detailed clinical and pathological review, aided by FISH and NGS studies, this series of cases also allow us to examine the new provisional entity of myeloid/lymphoid neoplasm with eosinophilia and rearrangement of $\mathrm{t}(8 ; 9)(\mathrm{p} 22 ; \mathrm{p} 24.1) / P C M 1-J A K 2$ and $\mathrm{t}$ $(9 \mathrm{p} 24.1 ; \mathrm{v}) / J A K 2$ variants.

We first questioned if the presence of a $J A K 2$ fusion could be assumed in cases with $\mathrm{t}(8 ; 9)(\mathrm{p} 22 ; \mathrm{p} 24)$ or in cases with $\mathrm{t}(9 \mathrm{p} 24 ; \mathrm{v})$. Of note, a significant proportion of cases of $\mathrm{t}$ $(8 ; 9)(\mathrm{p} 22 ; \mathrm{p} 24)$ reported to date were based on karyotyping alone, with no FISH or molecular confirmation of JAK2 involvement. From our initial search including all cases with 9p24 abnormalities, we detected JAK2 rearrangement in all cases with $\mathrm{t}(8 ; 9)(\mathrm{p} 22 ; \mathrm{p} 24.1)$ tested $(11 / 11)$. However, a JAK2 rearrangement was only found in about a quarter of the cases with $\mathrm{t}(9 \mathrm{p} 24 ; \mathrm{v})$. Of note, because PCM1-JAK2 FISH was only successful on cultured cells, we could perform it in only two cases, which did confirm the presence of PCM1-JAK2 fusion in both cases. Of note, published cases in which PCM1-JAK2 fusion was investigated either by reverse transcription-PCR or FISH revealed the presence of PCM1-JAK2 fusions in all tested cases of myeloid neoplasms with $\mathrm{t}(8 ; 9)(\mathrm{p} 22 ; \mathrm{p} 24.1)[5,12,13]$. Our results suggest that cases with $\mathrm{t}(8 ; 9)(\mathrm{p} 22 ; \mathrm{p} 24)$ demonstrated on a conventional karyotype may be assumed to have JAK2 rearrangement, most likely with $P C M 1$ based on prior reports $[5,12,13]$ and our findings. In contrast, it is absolutely necessary to perform FISH, RT-PCR, or RNA sequencing in cases with $\mathrm{t}(9 \mathrm{p} 24 ; \mathrm{v})$ to confirm a $J A K 2$ rearrangement. It is noteworthy that $J A K 2$ is located at the terminal band of the short arm of chromosome 9 . Since the $t$ (9p24.1;v) only involves a small fragment of chromosome $9 \mathrm{p}$, this rearrangement could be cryptic and hence missed by conventional chromosomal analysis. This would be especially problematic if the partner gene is also located on the terminal part of the chromosomal arm. Furthermore, a $J A K 2$ rearrangement due to an insertion would be extremely difficult to visualize on chromosomal analysis. Thus, these variant $J A K 2$ rearrangements may be under-recognized. As RNA sequencing is being increasingly used in clinical practice, more cases with JAK2 rearrangements might be identified, especially those with variant partner genes. Nonetheless, JAK2 rearrangements appear to be extremely rarely in hematopoietic neoplasms at the time of initial diagnosis. Only 13 cases were identified from seven large medical centers over a median 10 year period, yielding an estimated frequency of $<0.1 \%$ of all myeloid neoplasms.

The most common presentation was MPN, including six patients with $\mathrm{t}(8 ; 9)(\mathrm{p} 22 ; \mathrm{p} 24.1) / P C M 1-J A K 2$ and one patient with $\mathrm{t}(9 ; 22)(\mathrm{p} 24.1 ; \mathrm{q} 11.2) / B C R-J A K 2$. These MPNs did 
Fig. 3 Bone marrow (BM) and lymph node morphological findings of case \#5 (chronic phase of a myeloproliferative neoplasm with extramedullary disease infiltration $)$. a $(\times 40) \mathrm{BM}$ core biopsy section shows hypercellularity with two immature erythroid islands; megakaryocytes are present with unremarkable morphology; b (×40) lymph node biopsy shows large aggregates of early erythroid precursors, similar to that seen in the bone marrow
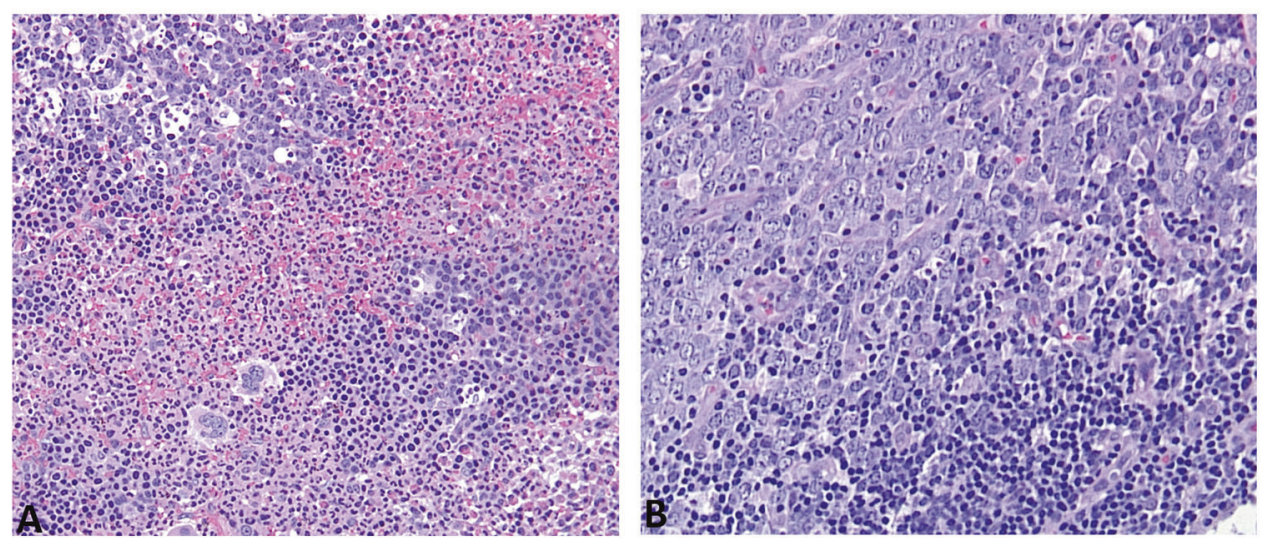

share a number of characteristic features, similar to what have been reported in the literature [3,5, 14, 15], including variable degrees of eosinophilia, either in $\mathrm{PB}$ or $\mathrm{BM}$ or both; frequent marrow fibrosis, and large aggregates of immature erythroid precursors. Hepatosplenomegaly (6/7 patients) and lymphadenopathy (3 of 5 with information available) were very common clinical findings. Three patients had lymph node biopsies; all three showed extramedullary involvement by a MPN, with large clusters or aggregates of immature erythroid precursors that raised the concern of pure erythroid leukemia transformation in one case. In addition to these MPN, one patient was diagnosed with MDS and one CMML. These two cases showed less distinct clinicopathological features as compared to MPNs. Specifically, there was no PB eosinophilia, although both patients did show mild eosinophilia in the BM. In addition, there were no large immature erythroid aggregates identified, but the MDS case did show small clusters of immature erythroid cells in the BM. MF3 fibrosis was present in the CMML case. To our knowledge, a presentation of myeloid neoplasm with $\mathrm{t}(8 ; 9)(\mathrm{p} 22 ; \mathrm{p} 24.1)$ as MDS has not been previously reported and thus it is unclear if this case should be included in the provisional group of myeloid/lymphoid neoplasms with PCM1-JAK2 as currently defined in the 2016 WHO Classification. Of these nine patients with myeloid neoplasms, only one was treated with JAK2 inhibitor. The survivals of these patients were variable. For patients with a diagnosis of MPN in chronic phase, the disease appeared to be indolent, similar to what has been reported in the literature $[3,5,14,15]$. However, the one patient with MPN apparently in accelerated/blast phase had an aggressive disease course, similar to other advanced phase MPN [16].

B-ALL was the second most common presentation among patients with $J A K 2$ rearrangement, including two with $\mathrm{t}(8 ; 9)(\mathrm{p} 22 ; \mathrm{p} 24.1)$ and two with $\mathrm{t}(9 \mathrm{p} 24 ; \mathrm{v})$. Similar to reported B-ALL cases with $\mathrm{t}(8 ; 9)(\mathrm{p} 22 ; \mathrm{p} 24.1)$ [3, 5, 17-19], none of the four cases showed eosinophilia in PB or BM. It is known that JAK2 rearrangement in B-ALL is one of the genetic alterations in Ph-like B-ALL with activated JAKSTAT pathway. Up to date, at least 20 different partner genes with JAK2 fusions have been identified in Ph-like $\mathrm{B}$ ALL [20-22]. The most frequent partner genes include SSBP2, PAX5, RFX3, USP25, and ZNF274. In cases without a documented MPN and lack of eosinophilia, such cases are suggested to be appropriately considered as Ph-like BALL [23]. Of the two B-ALL patients with $\mathrm{t}(8 ; 9)(\mathrm{p} 22$; p24.1), although neither patient had a prior history of a chronic myeloid neoplasm, we demonstrated JAK2 rearrangement in the background neutrophils and erythroid cells in addition to the B-lymphoblasts in one case by combined morphology-FISH and another by the presence of a high percentage of $J A K 2$ rearrangement in a BM sample (many in segmented neutrophils) with only minimal residual B-ALL. This data suggest the possibility that these two BALL cases with $\mathrm{t}(8 ; 9)(\mathrm{p} 22 ; \mathrm{p} 24.1)$ may represent Blymphoblastic crisis of an undocumented chronic myeloid neoplasm. These features are reminiscent of myeloid/lymphoid neoplasm with $F G F R 1$ rearrangement [24, 25], in which the genetic lesion affects pluripotent stem cells and may involve myeloid, lymphoid or both lineages, concomitantly or sequentially, leading to complex and heterogeneous clinical manifestations. On the other hand, one BALL with $\mathrm{t}(5 ; 9)(\mathrm{q} 14 ; \mathrm{p} 24.1)$ possibly SSBP2-JAK2 and another $\mathrm{t}(5 ; 9)(\mathrm{q} 11.2 ; \mathrm{p} 24.1)(J A K 2$ with an unknown partner gene) both showed no evidence of $J A K 2$ rearrangement at remission, suggesting that $J A K 2$ rearrangement was limited to the B-lymphoblasts. Our findings support the 2017 WHO Classification inclusion of cases presenting as B-ALL with $t$ $(8 ; 9)(\mathrm{p} 22 ; \mathrm{p} 24.1)$ within the group of myeloid/lymphoid neoplasms with $\mathrm{t}(8 ; 9)(\mathrm{p} 22 ; \mathrm{p} 24.1) / P C M 1 / J A K 2$. Conversely, B-ALL with $\mathrm{t}(9 \mathrm{p} 24 ; \mathrm{v}) / J A K 2$ be more appropriately classified as Ph-like B-ALL, as suggested in the WHO Classification. Gene expression profiling study may be useful in evaluating the relationship of these cases to other Ph-like B-ALL with activated JAK-STAT pathway. In addition, the classification could be further clarified by the presence or absence of a previous history of an MPN, or by 

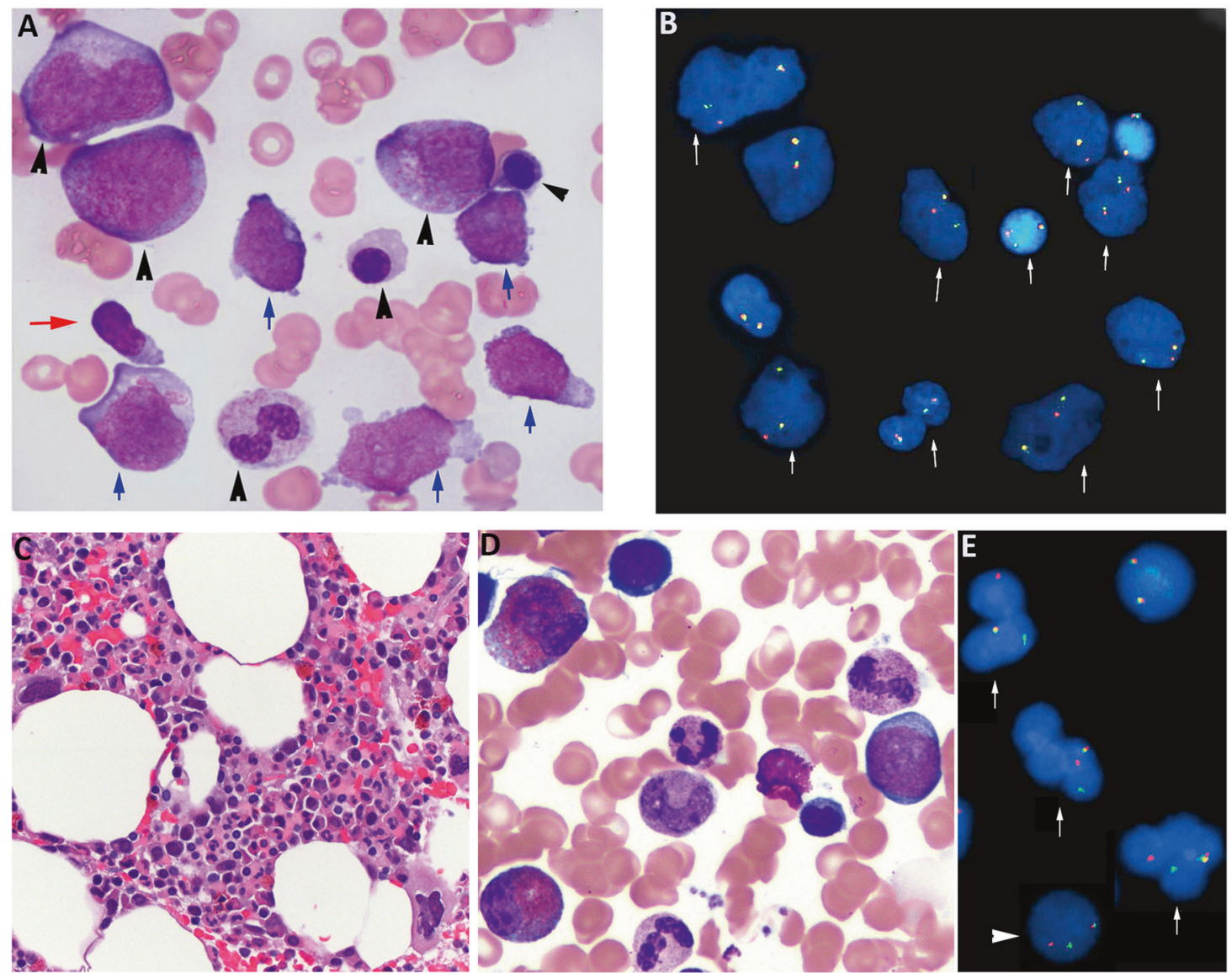

Fig. 4 Two cases of B-lymphoblastic leukemia (B-ALL) with $\mathrm{t}(8 ; 9)$ (p22;p24)/PCM1-JAK2. a, b Patient \#9, day 21 post induction chemotherapy bone marrow (BM) showed persistent B-ALL with $28 \%$ blasts. a $(\times 100)$ Wright-Giemsa stain, blue arrows point at blasts, black arrow heads point at erythrocytes, granulocytes and their precursors; and the red arrow points at a lymphocyte; b FISH using JAK2 dualcolor break-apart probe was performed and mapped back to the Wright-Giemsa stained slide A. While the lymphocyte shows two intact signals (no JAK2 rearrangement); a split signal pattern (1 red 1 green, marked with arrows) indicating $J A K 2$ rearrangement is detected

evidence of MPN in a post-treatment remission marrow, including demonstration of persistent $J A K 2$ rearrangement. This approach would be analogous to distinguishing a Blymphoblastic crisis of chronic myeloid leukemia (CML) from de novo $\mathrm{Ph}+\mathrm{B}$-lymphoblastic leukemia.

Mutation data are scarce in cases with 9q24.1/JAK2 rearrangement, largely due to the unavailability of NGS testing in earlier reported cases. In our cohort, six patients had NGS performed. All three cases of MPNs with $t(8 ; 9)$ (p22;p24.1) showed no mutations. In contrast, mutations were detected in the case of MDS and in two B-ALL with $t$ $(8 ; 9)(\mathrm{p} 22 ; \mathrm{p} 24.1)$. None of mutations were specific or unique. The most common mutations were $\operatorname{ASXL1}(n=2)$ and TET2 $(n=2)$. One patient with B-ALL and $\mathrm{t}(8 ; 9)(\mathrm{p} 22$; p24.1) showed a BCOR mutation. Interestingly, a recent study that performed NGS on seven patients with PCM1$J A K 2$ detected only a BCOR mutation in one patient (14\%) not only in lymphoblasts but also in erythrocytes, granulocytes and their precursors. c-e Patient \#10, 7 months post induction chemotherapy for B-ALL and in the course of consolidation. $\mathbf{c}(\times 20) \mathrm{BM}$ showed a hypercellularity for age (69 years), with normal-appearing megakaryocytes; $\mathbf{d}(\times 100)$ blasts were not increased, and there was no significant dyserythropoiesis or dysgranulopoiesis. Some eosinophil precursors were seen; e FISH using JAK2 dual-color break-apart probe revealed $J A K 2$ rearrangement in $41 \%$ cells, many in segmented neutrophils (marked by white arrow)

but no mutations in other six patients [26]. The additional somatic mutations might contribute to the disease heterogeneity observed in this cytogenetically defined entity; though further study is needed to determine their significance.

In summary, we show that hematopoietic neoplasms with 9p24/JAK2 rearrangement are extremely rare, and a large proportion of the cases are $\mathrm{t}(8 ; 9)(\mathrm{p} 22 ; \mathrm{p} 24.1) / P C M 1-J A K 2$. The neoplasms with $\mathrm{t}(8 ; 9)(\mathrm{p} 22 ; \mathrm{p} 24.1) / P C M 1-J A K 2$ frequently present as myeloproliferative neoplasms with variable eosinophilia, and characteristic clinical and BM findings, but may also present as B-ALL, a manifestation of B-lymphoblastic crisis of an underlying chronic myeloid neoplasm. Cases rarely present as MDS and CMML, which show less distinctive clinicopathologic characteristics. It is debatable if hematopoietic neoplasms with $\mathrm{t}(8 ; 9)(\mathrm{p} 22$; p24.1)/PCM1-JAK2, a provisional entity under "the 
myeloid/lymphoid neoplasm with eosinophilia and a specific gene rearrangement" should only include cases of a MPN presentation that may present in chronic, accelerated or blast phase, or analogous to other members of the group (PDBGFA, PBGFRB and FGFRI) should be a molecularlydefined disease. It is noteworthy that absence of eosinophilia and heterogeneous disease presentations are also seen in myeloid/lymphoid neoplasms with PDBGFA, PBGFRB and FGFR1 rearrangement. Cases of JAK2 with variant partner genes are even rarer. While cases of $\mathrm{t}(9 ; 22)(\mathrm{p} 24.1$; $\mathrm{q} 11.2) / B C R-J A K 2$ and $\mathrm{t}(9 ; 12)(\mathrm{p} 24.1 ; \mathrm{p} 13) / E T V 6-J A K 2$, or cases with a MPN-like presentation may have features similar to $\mathrm{t}(8 ; 9)(\mathrm{p} 22 ; \mathrm{p} 24.1) / P C M 1-J A K 2$ myeloid neoplasms, cases with a de novo B-ALL presentation may be more appropriately classified within the Ph-like (BCR$A B L 1$-like) B-ALL category. However, due to the small number of such cases in our cohort, further study is needed to determine their optimal classification.

\section{Compliance with ethical standards}

Conflict of interest The authors declare that they have no conflict of interest.

\section{References}

1. Ihle JN, Gilliland DG. Jak2: normal function and role in hematopoietic disorders. Curr Opin Genet Dev. 2007;17:8-14.

2. Santos FP, Verstovsek S. JAK2 inhibitors: what's the true therapeutic potential? Blood Rev. 2011;25:53-63.

3. Bain BJ, Ahmad S. Should myeloid and lymphoid neoplasms with PCM1-JAK2 and other rearrangements of JAK2 be recognized as specific entities? Br J Haematol. 2014;166:809-17.

4. Stewart K, Carstairs KC, Dube ID, Keating A. Neutrophilic myelofibrosis presenting as Philadelphia chromosome negative BCR non-rearranged chronic myeloid leukemia. Am J Hematol. 1990;34:59-63.

5. Reiter A, Walz C, Watmore A, et al. The $\mathrm{t}(8 ; 9)(\mathrm{p} 22 ; \mathrm{p} 24)$ is a recurrent abnormality in chronic and acute leukemia that fuses PCM1 to JAK2. Cancer Res. 2005;65:2662-7.

6. Rumi E, Milosevic JD, Selleslag D, et al. Efficacy of ruxolitinib in myeloid neoplasms with PCM1-JAK2 fusion gene. Ann Hematol. 2015;94:1927-8.

7. Bain BJ, Horny H-P, Arber DA, Tefferi A, Hasserjian RP. Myeloid/lymphoid neoplasms with eosinophilia and rearrangements of PDGFRA, PDGFRB or FGFR1, or with PCM1-JAK2. In: Swerdlow SH, Campo E, Harris NL, Jaffe ES, Pileri SA, Stein H, Thiele J, editors. WHO Classification of Tumours of Haematopoietic and Lymphoid Tissues Revised. 4th edn. Lyon: International Agency for Research on Cancer (IARC); 2017. p. 72-9.

8. Tang G, Goswami RS, Liang CS, et al. Isolated del $(5 q)$ in patients following therapies for various malignancies may not all be clinically significant. Am J Clin Pathol. 2015;144:78-86.

9. Thiele J, Kvasnicka HM, Facchetti F, et al. European consensus on grading bone marrow fibrosis and assessment of cellularity. Haematologica. 2005;90:1128-32.
10. Wang SA, Tam W, Tsai AG, et al. Targeted next-generation sequencing identifies a subset of idiopathic hypereosinophilic syndrome with features similar to chronic eosinophilic leukemia, not otherwise specified. Mod Pathol. 2016;29:854-64.

11. Wang SA, Hasserjian RP, Tam W, et al. Bone marrow morphology is a strong discriminator between chronic eosinophilic leukemia, not otherwise specified and reactive idiopathic hypereosinophilic syndrome. Haematologica. 2017;102:1352-60.

12. Schwaab J, Knut M, Haferlach C, et al. Limited duration of complete remission on ruxolitinib in myeloid neoplasms with PCM1-JAK2 and BCR-JAK2 fusion genes. Ann Hematol. 2015;94:233-8.

13. Rumi E, Milosevic JD, Casetti I, et al. Efficacy of ruxolitinib in chronic eosinophilic leukemia associated with a PCM1-JAK2 fusion gene. J Clin Oncol. 2013;31:e269-71.

14. Murati A, Gelsi-Boyer V, Adelaide J, et al. PCM1-JAK2 fusion in myeloproliferative disorders and acute erythroid leukemia with $\mathrm{t}$ $(8 ; 9)$ translocation. Leukemia. 2005;19:1692-6.

15. Bousquet M, Quelen C, De Mas V, et al. The t( $8 ; 9)(\mathrm{p} 22 ; \mathrm{p} 24)$ translocation in atypical chronic myeloid leukaemia yields a new PCM1-JAK2 fusion gene. Oncogene. 2005;24:7248-52.

16. Hidalgo Lopez JE, Carballo-Zarate A, Verstovsek S, et al. Bone marrow findings in blast phase of polycythemia vera. Ann Hematol. 2018;97:425-34.

17. Peeters P, Raynaud SD, Cools J, et al. Fusion of TEL, the ETSvariant gene 6 (ETV6), to the receptor-associated kinase JAK2 as a result of $\mathrm{t}(9 ; 12)$ in a lymphoid and $\mathrm{t}(9 ; 15 ; 12)$ in a myeloid leukemia. Blood. 1997;90:2535-40.

18. Zhou MH, Gao L, Jing Y, et al. Detection of ETV6 gene rearrangements in adult acute lymphoblastic leukemia. Ann Hematol. 2012;91:1235-43.

19. Tirado CA, Chen W, Huang LJ, et al. Novel JAK2 rearrangement resulting from a $\mathrm{t}(9 ; 22)(\mathrm{p} 24 ; \mathrm{q} 11.2)$ in B-acute lymphoblastic leukemia. Leuk Res. 2010;34:1674-6.

20. Roberts KG, Gu Z, Payne-Turner D, et al. High Frequency and Poor Outcome of Philadelphia Chromosome-Like Acute Lymphoblastic Leukemia in Adults. J Clin Oncol. 2017;35:394-401.

21. Reshmi SC, Harvey RC, Roberts KG, et al. Targetable kinase gene fusions in high-risk B-ALL: a study from the Children's Oncology Group. Blood. 2017;129:3352-61.

22. Roberts KG, Li Y, Payne-Turner D, et al. Targetable kinaseactivating lesions in Ph-like acute lymphoblastic leukemia. N Engl J Med. 2014;371:1005-15.

23. Borowitz MJ, Chan JKC, Downing JR, Le Beau MM, Arber D. Blymphoblasticleukemia/lymphoma with recurrent genetic abnormalities. In: Swerdlow SH, CE, Harris NL, Jaffe ES, Pileri SA, Stein H, Thiele J, editors. WHO classification of Tumors of Haematopoietic and Lymphoid Tissue. Lyon: IARC; 2017.

24. Keane C, Henden A, Mills T, Wood P. Myeloid and lymphoid neoplasm with FGFR1 abnormality. $\mathrm{Br} \mathrm{J}$ Haematol. 2009; $145: 440$.

25. Wang W, Tang G, Kadia T, et al. Cytogenetic Evolution Associated With Disease Progression in Hematopoietic Neoplasms With $\mathrm{t}(8 ; 22)(\mathrm{p} 11 ; \mathrm{q} 11) / \mathrm{BCR}-F G F R 1$ Rearrangement. J Natl Compr Canc Netw. 2016;14:708-11.

26. Baer C, Muehlbacher V, Kern W, Haferlach C, Haferlach T. Molecular genetic characterization of myeloid/lymphoid neoplasms associated with eosinophilia and rearrangement of PDGFRA, PDGFRB, FGFR1 or PCM1-JAK2. Haematologica. 2018. 